\title{
THEORETICAL INVESTIGATION OF THE COMPLETE PHASE DIAGRAM OF THE $\mathrm{SmMn}_{2} \mathrm{Ge}_{2}$ METAMAGNET*
}

\author{
P. Polaszek AND Z. ONYSZKiEwicz \\ Magnetism Theory Division, Institute of Physics, A. Mickiewicz University \\ Matejki 48/49, 60-769 Poznań, Poland \\ (Received June 14, 1993; in final form October 20, 1993)
}

\begin{abstract}
A two-sublattice Ising model with spatial four-spin and six-spin anisotropy added in order to describe $\mathrm{SmMn}_{2} \mathrm{Ge}_{2}$ metamagnet is discussed. The complete phase diagrams for this metamagnet as a function of hydrostatic pressure are obtained.
\end{abstract}

PACS numbers: $75.30 . \mathrm{Kz}$

\section{Introduction}

The intermetallic compound $\mathrm{SmMn}_{2} \mathrm{Ge}_{2}$ represents a class of very interesting temperature-induced metamagnets (TIM) [1-5]. With decreasing temperature (without magnetic field $H$ and under normal hydrostatic pressure $p$ ) the following sequence of phases is observed: paramagnetic $(P)$, ferromagnetic $(F)$ in the temperature region $T_{\mathrm{FA}}<T<T_{\mathrm{C}}$, antiferromagnetic (A) which becomes stable for $T_{\mathrm{AF}}<T<T_{\mathrm{FA}}$ and re-entrant ferromagnetic which appears below $T_{\mathrm{AF}}$. The Curie temperature $T_{\mathrm{C}}=341 \mathrm{~K}$, temperature of metamagnetic transitions $T_{\mathrm{FA}}=153 \mathrm{~K}$ and $T_{\mathrm{AF}}=106.5 \mathrm{~K}[4]$.

During the last decade some progress has been made in the understanding of metamagnetic phenomena. To describe theoretically the behaviour of TIM, the model spin Hamiltonian has been mostly used. In this paper we apply the Ising model with spatial four-spin and six-spin anisotropy [6] added in order to describe the complete phase diagram of $\mathrm{SmMn}_{2} \mathrm{Ge}_{2}$ in the space of the parameters: $H, p$ and $T$. As our basic assumption we take the parameters of the Hamiltonian to be linear functions of pressure $p$. This assumption is justified by the experimental fact that $T_{\mathrm{C}}$ is a linear function of hydrostatic pressure in a wide range of its variation $[4,5]$.

${ }^{*}$ This work was sponsored by the project 223519102 of the Committee for Scientific Research. 


\section{Hamiltonian and molecular field approximation (MFA)}

We take the following Hamiltonian as the basic assumption $\left(s=\frac{1}{2}\right)$ :

$$
\begin{aligned}
& \mathcal{H}=-\frac{1}{2} \sum_{f_{\neq \neq f^{\prime}}} I_{1 f f^{\prime}}(p) S_{f}^{Z} S_{f^{\prime}}^{Z}-\frac{1}{2} \sum_{\boldsymbol{g}_{\neq \neq} \boldsymbol{g}^{\prime}} I_{1 \boldsymbol{g} g^{\prime}}(p) S_{\boldsymbol{g}^{\prime}}^{Z} S_{\boldsymbol{g}^{\prime}}^{Z} \\
& +\sum_{\boldsymbol{f} \neq \boldsymbol{g}} I_{2 \boldsymbol{f} \boldsymbol{g}}(p) S_{f}^{Z} S_{\boldsymbol{g}}^{Z}-g \mu_{\mathrm{B}} H\left(\sum_{\boldsymbol{f}} S_{f}^{Z}+\sum_{\boldsymbol{g}} S_{\boldsymbol{g}}^{Z}\right) \\
& +\frac{1}{2} \sum_{f g f^{\prime} f^{\prime \prime}} D_{f g f^{\prime} f^{\prime \prime}}(p) S_{f}^{Z} S_{g}^{Z} S_{f^{\prime}}^{Z} S_{f^{\prime \prime}}^{Z} \\
& +\frac{1}{2} \sum_{f g f^{\prime} f^{\prime \prime} f^{\prime \prime \prime} f^{\prime \prime \prime \prime}} K_{f g f^{\prime} f^{\prime \prime} f^{\prime \prime \prime} f^{\prime \prime \prime}}(p) S_{f}^{Z} S_{g}^{Z} S_{f^{\prime}}^{Z} S_{f^{\prime \prime}}^{Z} S_{f^{\prime \prime \prime}}^{Z} S_{f^{\prime \prime \prime \prime}}^{Z} \\
& +\frac{1}{2} \sum_{f g^{\prime} g^{\prime \prime}} D_{f g g^{\prime} g^{\prime \prime}}(p) S_{f}^{Z} S_{g}^{Z} S_{g^{\prime}}^{Z} S_{g^{\prime \prime}}^{Z}
\end{aligned}
$$

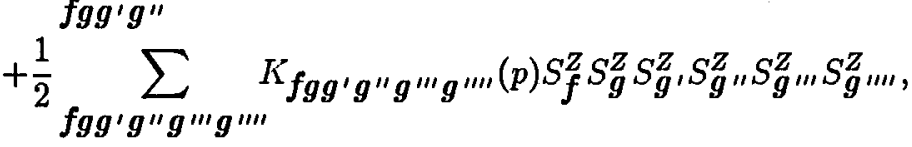

where $\sum$ stands for a summation over different lattice points (nearest neighbours). The indices $f$ and $g$ refer to spin sites in the sublattices 1 and 2, respectively. This model is a fusion of models proposed in [6] (six-spin terms) and in [7] (term with magnetic field). Now MFA enables us to study thermodynamic properties of model (1). Applying the variation principle for the trial free energy $\widetilde{\mathcal{F}}$ we write

$$
\mathcal{F} \leq \widetilde{\mathcal{F}}=-\frac{1}{\beta} \ln \operatorname{Tr}\left(\exp \left(-\beta \mathcal{H}_{0}\right)\right)+\left\langle\mathcal{H}-\mathcal{H}_{0}\right\rangle_{0}
$$

where

$$
\begin{aligned}
& \langle\cdots\rangle_{0}=\operatorname{Tr}\left(\cdots \rho_{0}\right), \\
& \rho_{0}=\left\{\operatorname{Tr}\left[\exp \left(-\beta \mathcal{H}_{0}\right)\right]\right\}^{-1} \exp \left(-\beta \mathcal{H}_{0}\right)
\end{aligned}
$$

and $\beta=\left(k_{\mathrm{B}} T\right)^{-1}$. The symbol $\mathcal{H}_{0}$ denotes a trial Hamiltonian which is specified as follows:

$$
\mathcal{H}_{0}=-\lambda_{1} \sum_{f} S_{f}^{Z}-\lambda_{2} \sum_{\boldsymbol{g}} S_{\boldsymbol{g}}^{Z}
$$

From the minimum conditions for the trial free energy $\tilde{\mathcal{F}}$ :

$$
\frac{\partial \widetilde{\mathcal{F}}}{\partial \lambda_{1}}=\frac{\partial \tilde{\mathcal{F}}}{\partial \lambda_{2}}=0
$$

we can determine the variational parameters $\lambda_{1}$ and $\lambda_{2}$ (molecular fields):

$$
\begin{aligned}
\lambda_{1}= & B(p)\left\langle S_{f}^{Z}\right\rangle-G(p)\left\langle S_{g}^{Z}\right\rangle-\frac{1}{2} D(p)\left\langle S_{g}^{Z}\right\rangle\left(3\left\langle S_{f}^{Z}\right\rangle^{2}+\left\langle S_{g}^{Z}\right\rangle^{2}\right) \\
& -\frac{1}{2} C(p)\left\langle S_{g}^{Z}\right\rangle\left(5\left\langle S_{f}^{Z}\right\rangle^{4}+\left\langle S_{g}^{Z}\right\rangle^{4}\right)+g \mu_{\mathrm{B}} H
\end{aligned}
$$




$$
\begin{aligned}
\lambda_{2}= & B(p)\left\langle S_{g}^{Z}\right\rangle-G(p)\left\langle S_{f}^{Z}\right\rangle-\frac{1}{2} D(p)\left\langle S_{f}^{Z}\right\rangle\left(3\left\langle S_{g}^{Z}\right\rangle^{2}+\left\langle S_{f}^{Z}\right\rangle^{2}\right) \\
& -\frac{1}{2} C(p)\left\langle S_{f}^{Z}\right\rangle\left(5\left\langle S_{g}^{Z}\right\rangle^{4}+\left\langle S_{f}^{Z}\right\rangle^{4}\right)+g \mu_{\mathrm{B}} H
\end{aligned}
$$

where

$$
\begin{aligned}
& N B(p)=\sum_{\boldsymbol{f} \neq \boldsymbol{f}^{\prime}} I_{1 f f^{\prime}}(p), \quad N G(p)=\sum_{\boldsymbol{f} \neq \boldsymbol{g}} I_{2 f \boldsymbol{g}}(p), \\
& N D(p)=\sum_{f g f^{\prime} f^{\prime \prime}} D_{f g f^{\prime} f^{\prime \prime}}(p)=\sum_{f g g^{\prime} g^{\prime \prime}} D_{f g g^{\prime} g^{\prime \prime}}(p), \\
& N C(p)=\sum K_{f g f^{\prime} f^{\prime \prime} f^{\prime \prime \prime} f^{\prime \prime \prime}}(p)=\sum \sum \\
& f g f^{\prime} f^{\prime \prime} f^{\prime \prime \prime} f^{\prime \prime \prime \prime}
\end{aligned}
$$

( $N$ is the number of sites in one sublattice).

In a simple way we can find

$$
\left\langle S_{f}^{Z}\right\rangle=\frac{1}{2} \tanh \left(\frac{1}{2} \beta \lambda_{1}\right), \quad\left\langle S_{g}^{Z}\right\rangle=\frac{1}{2} \tanh \left(\frac{1}{2} \beta \lambda_{2}\right) .
$$

These two above equations determine the temperature dependencies of magnetization of both sublattices

$$
\begin{aligned}
& t=\frac{x b(p)-y a(p)-\frac{1}{2} y d(p)\left(3 x^{2}+y^{2}\right)-\frac{1}{2} y c(p)\left(5 x^{4}+y^{4}\right)+h}{\operatorname{atanh}(x)} \\
& t=\frac{y b(p)-x a(p)-\frac{1}{2} x d(p)\left(3 y^{2}+x^{2}\right)-\frac{1}{2} x c(p)\left(5 y^{4}+x^{4}\right)+h}{\operatorname{atanh}(y)}
\end{aligned}
$$

where we used new symbols

$$
\begin{aligned}
& a(p)=\frac{G(p)}{B(p=0)}, b(p)=\frac{B(p)}{B(p=0)}, d(p)=\frac{D(p)}{4 B(p=0)}, c(p)=\frac{C(p)}{16 B(p=0)}, \\
& t=\frac{4 k_{\mathrm{B}} T}{B(p=0)}, \quad h=\frac{2 g \mu_{\mathrm{B}} H}{B(p=0)}, \quad x=2\left\langle S_{f}^{Z}\right\rangle, \quad y=2\left\langle S_{g}^{Z}\right\rangle .
\end{aligned}
$$

It is natural to think of variables $x$ and $y$ as measures of magnetization of sublattices 1 and 2 respectively. Moreover, we can write now the total magnetization

$$
m=\left\langle S_{f}^{Z}\right\rangle+\left\langle S_{g}^{Z}\right\rangle
$$

in the form

$$
m=\frac{x+y}{2},
$$

which is used in computer calculations.

If we take $f=(N B(p=0))^{-1} \widetilde{\mathcal{F}}$ we can also write

$$
\begin{aligned}
& f=\frac{1}{2} b(p)\left(x^{2}+y^{2}\right)-x y a(p)-\frac{3}{2} x y d(p)\left(x^{2}+y^{2}\right)-\frac{5}{2} x y c(p)\left(x^{4}+y^{4}\right) \\
& -t\left(2 \ln 2-\frac{1}{2} \ln \left(1-x^{2}\right)-\frac{1}{2} \ln \left(1-y^{2}\right)\right)
\end{aligned}
$$

and it is the free energy in MFA. 


\section{Phase diagrams}

Now we assume that the parameters $a(p), b(p), c(p)$ and $d(p)$ are linear functions of hydrostatic pressure $p$ :

$$
\begin{aligned}
& a(p)=a_{0}+a_{1} p+\mathrm{O}\left(p^{2}\right), \\
& b(p)=b_{0}+b_{1} p+\mathrm{O}\left(p^{2}\right), \\
& c(p)=c_{0}+c_{1} p+O\left(p^{2}\right), \\
& d(p)=d_{0}+d_{1} p+\mathrm{O}\left(p^{2}\right) .
\end{aligned}
$$

However, even in this case it is impossible to find an analytical solution of Eqs. (11) and (12) for any values of variable $h$ (magnetic field). Only in the simplest case $h=0$ (system without magnetic field) we can easily get much information about the phase diagram. That is made in [6] (expressions for the Curie and Néel temperature, coordinates of the bicritical (BC) point and approximate expressions for the points of metamagnetic transitions). In the antiferromagnetic phase for $h=0$ we have $x=-y$. From the mathematical point of view, Eqs. (11) and (12) become identical and finally we have to solve only one equation with one unknown value $x$. Moreover, in this case the temperature dependencies of $x$ and $y$ are monotonic functions and the problem can be easily numerically solved (the bisection method).

In general, we must solve the problem with two unknown values $x$ and $y$ (in two equations). When $h \neq 0$, in the F-phase $x$ and $y$ are still equal, but in the A-phase it is not true. The problem becomes much more difficult even if we want to find only the numerical solution for the magnetizations of both sublattices for any values of parameters: $t, p$ and $h$. First of all, the computer seeks the solution for $h=0$ in the low temperature region (for any pressure). Then it switches the magnetic field on (for which we want to have the phase diagram). In low temperatures the solution for $h=0$ is a good "starting point" to look for a solution for any other value of $h$. Therefore, the program can find $x$ and $y$ for $h \neq 0$ for low $t$. Then the computer simulates a slow increase in temperature and for any new $t$ it corrects $x$ and $y$. When the computer cannot find corrections for A-phase for new $t$ it takes this value as the Neel temperature.

When we know what values of magnetization fulfil Eqs. (11) and (12) in A- and F-phases for definite $t, p$ and $h$ it is easy to test which phase will be preferred. It is, of course, determined by the free energy. Our system will choose the phase with the lower value of free energy. If we repeat the above algorithm for different values of pressure we can get a phase diagram. We can repeat it for another magnetic field, therefore we can get phase diagrams for different magnetic fields and we can realize the task of this work.

\section{Results}

The results of the computer calculations are illustrated in Figs. 1 to 3 . They were obtained for the values of parameters listed in Table. Figure 1 shows the 

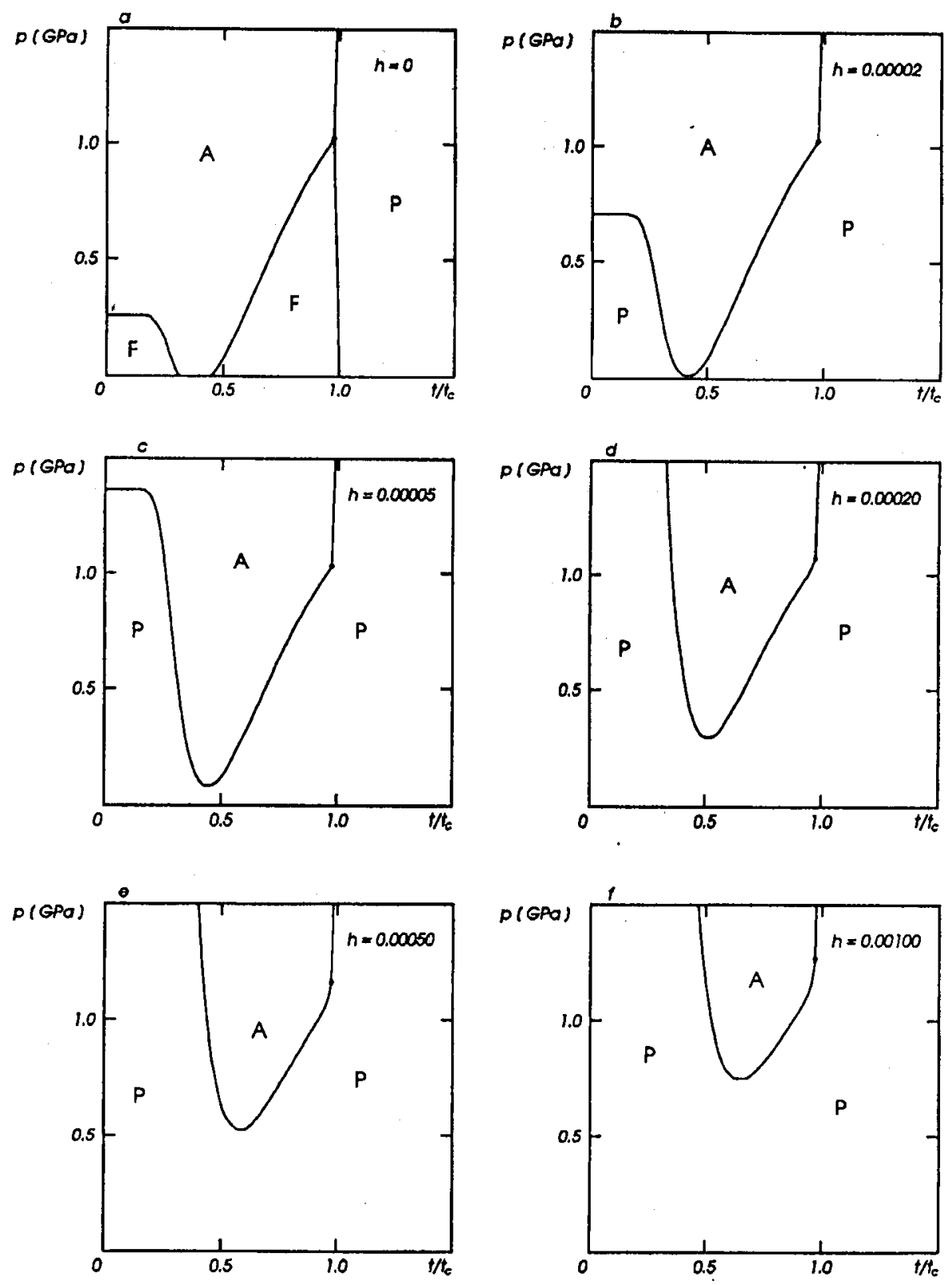

Fig. 1. Phase diagrams on the $(p, t)$ plane for different magnetic field $h$. Bicritical points are denoted by dots. 

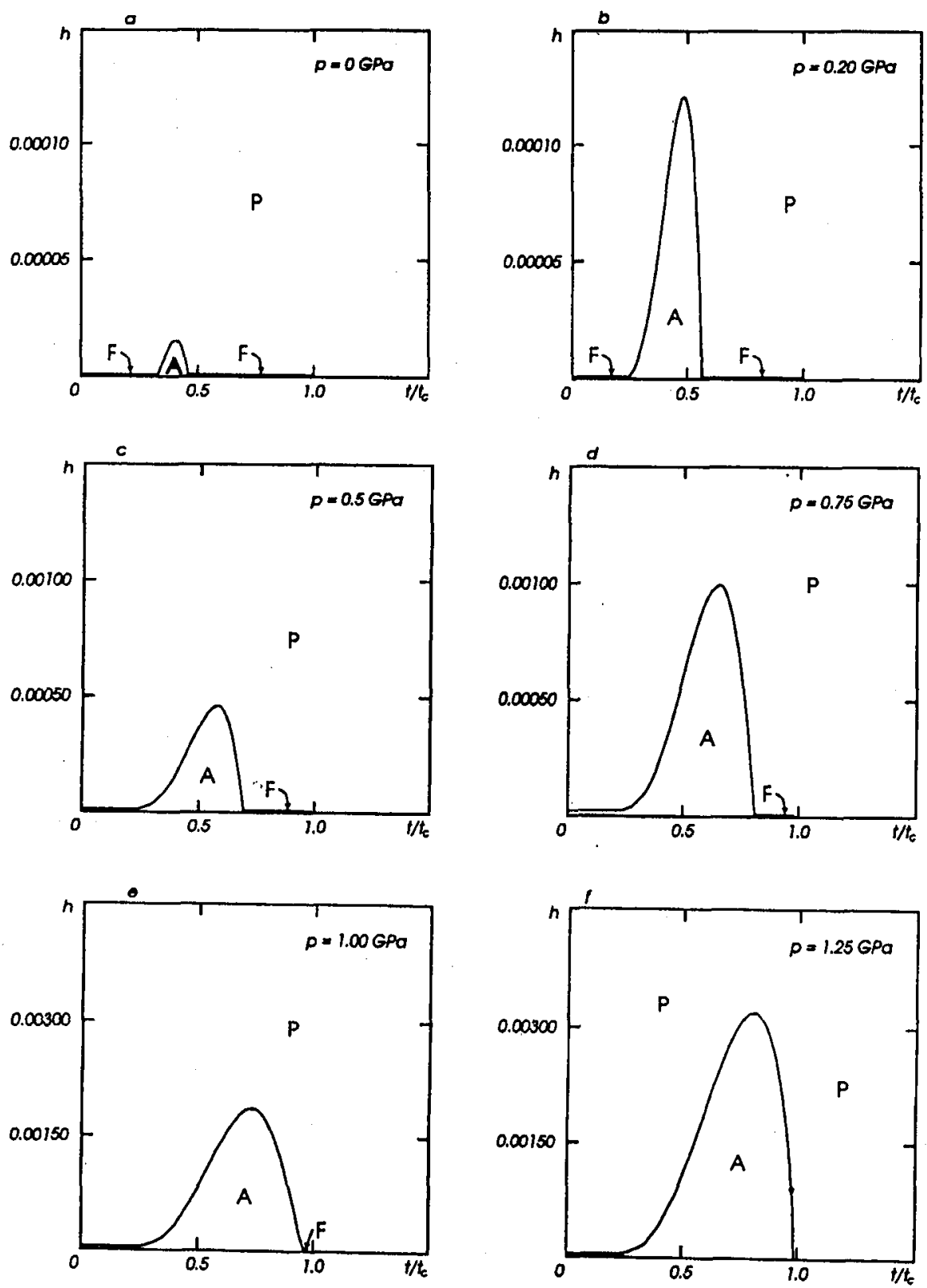

Fig. 2. Phase diagrams on the $(h, t)$ plane for different hydrostatic pressure $p$. Bicritical points are denoted by dots. 
a
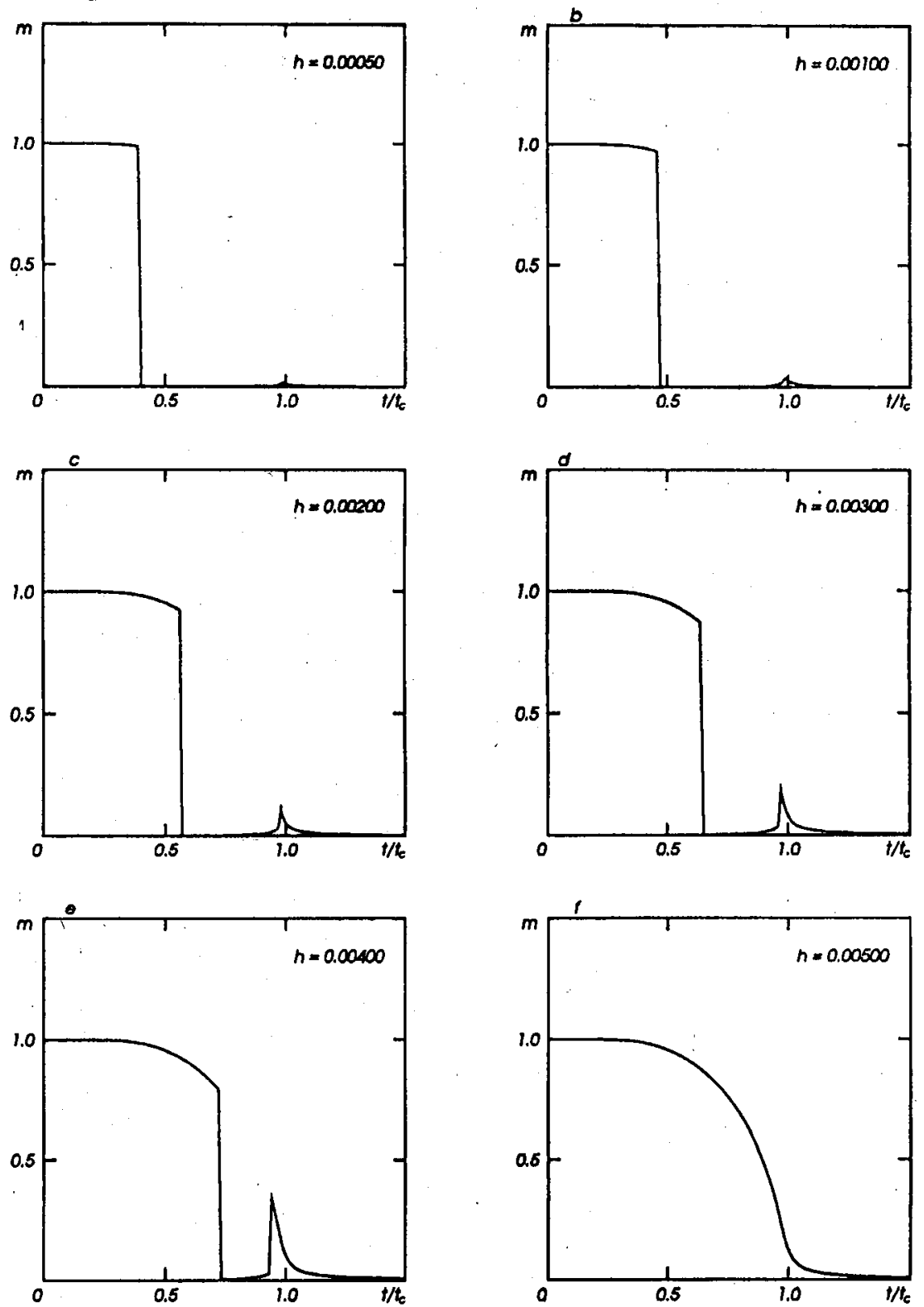

Fig. 3. The dependence of relative magnetization $m$ of $\mathrm{SmMn}_{2} \mathrm{Ge}_{2}$ TIM on relative temperature $t$ for $p=1.5 \mathrm{GPa}$ and for different magnetic field $h$. 


\section{TABLE}

The parameters used in the calculations of the phase diagrams of $\mathrm{SmMn}_{2} \mathrm{Ge}_{2}$ TIM.

\begin{tabular}{c|ll}
\hline \hline Parameter & & \\
\hline$a_{0}$ & -0.0240 & \\
$a_{1}$ & 0.0235 & $\mathrm{GPa}^{-1}$ \\
$b_{0}$ & 1.0 & \\
$b_{1}$ & 0.0 & $\mathrm{GPa}^{-1}$ \\
$c_{0}$ & -0.0256652551 & \\
$c_{1}$ & 0.014845 & $\mathrm{GPa}^{-1}$ \\
$d_{0}$ & 0.0496536302 & \\
$d_{1}$ & -0.0383 & $\mathrm{GPa}^{-1}$
\end{tabular}

phase diagram in the $(p, t)$ plane for different magnetic fields $h$. Figure 2 presents the phase diagram in the $(h, t)$ plane for different hydrostatic pressures $p$. Figure 3 presents the dependence of relative magnetization $m$ of TIM $\mathrm{SmMn}_{2} \mathrm{Ge}_{2}$ on relative temperature $t$ for $p=1.5 \mathrm{GPa}$ and for different magnetic fields $h$. It is easy to see that the value $0.00001 \mathrm{~J}$ of parameter $h$ is equivalent to the value $50 \mathrm{Gs}$ of real magnetic field $H$ (13). Computer did not calculate the coordinates of metamagnet transitions, but it only checked if for specific discrete values of $p, t$ and $h$, the Aor F-phase would be preferred. Therefore points of metamagnets transitions have a limited precision. The maximum error is: for $p-\Delta p=0.01 \mathrm{GPa}$, for $T-$ $\Delta T=0.01 T_{\mathrm{C}}$, for $h-\Delta h=0.000001 \mathrm{~J}$ (Fig. 2a, b), $\Delta h=0.00001 \mathrm{~J}$ (Fig. 2c, d) and $\Delta h=0.00003 \mathrm{~J}$ (Fig. 2e, f).

\section{Conclusions}

The results of our work are in agreement with the natural anticipation that the magnetic field prefers the F-phase as more energy-favourable. The magnetic field pushes out the A-phase into the large-pressure area of the phase diagram. It is important that the phase diagram calculated without the field is the same as in [6] and as in the experiments [5] (which is even more important). It would be interesting to realize an analogous experiment with magnetic field to verify our results.

A very interesting observation is that magnetization $m$ can increase with increasing temperature $t$ (see Figs. 3a to 3f, especially 3e). It is a consequence of the fact that magnetization of one sublattice falls down faster than the other, therefore their resultant can rise. 


\section{References}

[1] K.S.V.L. Narasimhan, V.U.S. Rao, R.L. Bergner, W.E. Wallace, J. Appl. Phys. 46, 4957 (1976).

[2] A. Szytuła, I. Szott, Solid State Commun. 40, 199 (1981).

[3] H. Fujii, T. Okamoto, T. Shigeoka, N. Iwata, Solid State Commun. 53, 715 (1985).

[4] M. Duraj, R. Duraj, A. Szytuła, Z. Tomkowicz, J. Magn. Magn. Mater. 73, 240 (1988).

[5] M. Duraj, R. Duraj, A. Szytuła, J. Magn. Magn. Mater. 79, 61 (1989).

[6] Z. Onyszkiewicz, Phys. Status Solidi B 163, K 111 (1991).

[7] Z. Onyszkiewicz, Acta Phys. Pol. A 77, 657 (1990). 